\title{
Monochromatic Infrared Photo Energy Versus Low Level Laser Therapy in Chronic Low Back Pain
}

\author{
Tarek Abdel Rahman Ali Ammar* \\ Department of Basic Science, Faculty of Physical Therapy, Cairo University, Cairo, Egypt
}

\section{*Correspondence to}

Tarek Abdel Rahman Ali Ammar,

PhD; Department of Basic Science,

Faculty of Physical Therapy, Cairo

University, Cairo, Egypt.

Tel: $+20-37617693$;

Fax: +20-37617692

Email: tarek.ali@alumni.twu.edu

Published online 27 October 2015

\begin{abstract}
Introduction: Low back pain (LBP) is the most common musculoskeletal disease. Monochromatic infrared photo energy (MIPE) and low level laser therapy (LLLT) are light modalities used to reduce pain and increase blood flow. The aim of this study was to compare the effects of the MIPE and LLLT in reducing functional disability and pain as well as improving lumbar range of motion (ROM) in patients with chronic LBP.

Methods: Seventy participants with LBP completed the program and were randomly assigned into 2 groups. Group $1(n=35)$ received MIPE and therapeutic exercises. Group $2(n=35)$ received LLLT and therapeutic exercises. Both groups received 2 visits per week for 6 weeks. Outcome measures were functional rating index (FRI), visual analogue scale (VAS) and modified-modified Schober test at baseline and after 6 weeks.

Results: There were statistically significant improvements in functional disability, pain and lumbar ROM $(P<.05)$ in each group. However, no significant differences were recorded between the groups $(P>.05)$.

Conclusion: Therefore, MIPE and LLLT may play a role in treating chronic LBP and there are no differences between the two modalities in improving functional disability, pain and lumbar ROM in patients with chronic LBP.

Keywords: Light; Back pain, low; Chronic; Monochromatic; Low level laser therapy; Pain.
\end{abstract}

\section{Introduction}

Low back pain (LBP) is a common disabling musculoskeletal disorder that is highly prevalent in the general population. LBP affects $60 \%-80 \%$ of individuals at some point in their lives, and most episodes of LBP resolve within weeks. However, LBP is also recurrent and chronic. Moreover, the costs of LBP have been escalating over the past 25 years. ${ }^{1}$ Physical therapy modalities are utilized to ameliorate pain and improve physical function in patients with LBP. Modalities include, but are not limited to, therapeutic exercises, transcutaneous electrical nerve stimulation, electrical stimulation, thermal modalities, low level laser therapy (LLLT), acupuncture, yoga and manipulation. ${ }^{2-9}$ Despite the wide variety of intervention options, physical therapy management of LBP might be still far from optimal.

Monochromatic infrared photo energy (MIPE) and LLTT are relatively new forms of light energy used to manage LBP. The monochromatic photo energy delivers MIPE with a wavelength of $890 \mathrm{~nm} .{ }^{10}$ The monochromatic light is emitted by an array of gallium aluminum arsenide diodes that are placed in the target skin. MIPE was used to decrease pain in diabetic peripheral neuropathy, knee osteoarthritis and restless leg syndrome. ${ }^{11-13}$ Researchers did not investigate intensively on using the MIPE in patients with musculoskeletal disorders and LBP.
LLLT is another form of light energy used in managing LBP. It is a noninvasive, monochromatic electromagnetic highly concentrated light beam and has a power output of less than $1 \mathrm{~W} / \mathrm{cm}^{2,14}$ LLLT has been used in various neuro-musculoskeletal disorders; however, its effectiveness is controversial. ${ }^{15}$ Some researchers suggested that it may relieve pain in temporomandibular disorders, shoulder myofascial pain syndrome and knee osteoarthritis. ${ }^{16-18}$ There has been a controversy about its effectiveness in LBP. ${ }^{19-20}$ Also, there may be limited evidence about the effectiveness of MIPE and LLLT on chronic LBP. The aim of the present study was to evaluate the short-term effectiveness of two modalities in the treatment of chronic LBP: MIPE and LLLT.

\section{Methods}

Design

This study was a randomized trial with participants randomly assigned to one of two treatment groups: (1) group 1 received the MIPE and therapeutic exercises or (2) group 2 received LLLT and therapeutic exercises. The examiner made group comparisons at the initial visit and after 6 weeks. The duration of the interventions was 6 weeks per participant, and each participant received 2 sessions per week. 


\section{Participants}

Participants who met the inclusion criteria were recruited from a physical therapy clinic. Patients were referred from a physician. A physical therapist conducted a thorough physical examination that included neurological testing, postural assessment, joint mobility, strength, etc. The inclusion criteria were symptomatic non-radiating LBP of more than 3 months duration between T12 and the gluteal fold and normal neurologic examination results.

Reasons for exclusion included a self-reported history of malignancy, vertigo, stroke, low back surgery with an implant, pregnancy and LBP accompanied by specific pathological conditions. All subjects signed a consent form permitting the use of their data for research purposes, and confidentiality was assured by the use of an anonymous coding system. Participants were asked to refrain from receiving other forms of physical therapy and analgesics during the study.

\section{Instrumentation}

The MIPE intervention was administered using the Anodyne $^{\circledR}$ Therapy System, model 480 (Anodyne Therapy, LLC, Tampa, Florida). The device consists of a base power unit and 8 therapy pads, each containing 60 gallium aluminum arsenide diodes. The area of anodyne light emitting diodes per therapy pads is $22.5 \mathrm{~cm}^{2}$, yielding a total treatment area of $180 \mathrm{~cm}^{2}$. The device delivers MIPE pulsed at $292 \mathrm{~Hz}$ with a wavelength of $890 \mathrm{~nm}$ through the diodes. The active unit provides $62.4 \mathrm{Joules} / \mathrm{cm}^{2}$ of energy density. ${ }^{21}$

The gallium-aluminum-arsenide (infrared laser) diode laser device (Chattanooga group, USA) was used with a power output of $100 \mathrm{~mW}$ and a wavelength of $850 \mathrm{~nm}$. The diameter of the laser beam at the treatment point was $1 \mathrm{~mm}$. The laser was set to deliver a continuous form of energy.

Outcomes Measures included the functional rating index (FRI) to measure functional disability. FRI is a new valid and reliable questionnaire to measure the magnitude of clinical change in spinal conditions. ${ }^{22}$ The examiners also used the visual analogue scale (VAS) to measure current pain intensity, average pain intensity over the past 2-3 days, and the worst level of pain experienced during the past 2-3 days. The mean of these scores was used to provide a measure of pain intensity. VAS is a valid and reliable tool to measure pain intensity. ${ }^{23}$ Measures also included the modifiedmodified Schober test to assess lumbar flexion and extension range of motion ( $\mathrm{ROM}){ }^{24}$

\section{Procedure}

Participants who met the inclusion criteria were randomly assigned to one of 2 groups. Randomization was done by a computer generated random table. Only one independent investigator, blinded to group allocation, conducted the testing procedures at baseline and after 6 weeks. After initial testing, participants began the treatment on the same day. Another licensed physical therapist performed all interventions with the participants in both groups. All participants received 2 sessions per week for 6 weeks. Group 1 received the MIPE for 30 minutes and therapeutic exercises for 20 minutes. Each subject sat comfortably in a quiet room at $21^{\circ} \mathrm{C}$. The skin of the treatment area was covered with plastic wrap as a barrier between the skin and the diodes to ensure compliance with infection control procedures. The therapy pads were put on either side of the lumbar spine. The energy setting on the device was preset at 10 bars for every patient, in accordance with the manufacturer's recommendations. The diodes and plastic wrap were removed at the end of the treatment session. Intervention with the MIPE was followed by therapeutic exercises for 20 minutes. The exercise program started with stretching routines for the lower limbs as well as the lower back. Then, strengthening exercises for abdominal and paravertebral muscles of the low back were gradually added to each session.

Group 2 received LLLT (wavelength $850 \mathrm{~nm}$, continuous wave, power $100 \mathrm{mV}$, dose $5 \mathrm{~J} /$ point). The diameter of the laser beam at the irradiated point was $1 \mathrm{~mm}$. Participants underwent irradiation for 90 seconds at 8 symmetric points along the lumbosacral spine (T12-S3). They also did the same therapeutic exercises undertaken by the participants in group 1.

The therapist educated all participants regarding the rationale for MIPE and LLLT. The therapist provided verbal and written instructions related to the proper method of exercise, and participants demonstrated to the treating physical therapist their ability to properly perform the prescribed exercises. All participants were instructed to exercise at home on the days that they did not go to the clinic, and the home program was monitored by asking the participants to record exercise using weekly self-reported exercise logs.

\section{Statistical Analysis}

All analyses were performed using the SPSS 11.5 for Windows software program. After assessing the normal distribution of the data, separate univariate analyses of covariance with the pretest scores as the covariates were performed to determine whether there were differences between the two groups on the post test scores of functional disability, pain and lumbar ROM. A Bonferroni approach was used to maintain the alpha level at $P<.05$. Differences with $P$ values $\leq .05$ were considered statistically significant. Continuous variables were summarized as means and standard deviations.

\section{Results}

Seventy-six patients with chronic LBP (48 women, 28 men) aged between $35-60$ years participated in the study. Participants with chronic LBP were randomly assigned into 2 groups. Three women and one man dropped out in group 1; whereas one man and one woman dropped out in group 2. Reasons for the drop outs included social reasons, lack of time and lack of interest. Therefore, seventy participants completed the study program. Group 1 consisted of 35 participants (14 men and 21 women). Group 2 
also consisted of 35 participants ( 12 men and 23 women). Patient flow through the study is shown in the CONSORT flow chart (Figure 1).

Baseline characteristics of the 70 participants who completed the study are given in Table 1. There were no statistically significant differences between the treatment groups in regard to age, onset of pain since first episode, and body mass index $(P>.05)$.

Mean values and standard deviations of the disability, pain intensity and lumbar ROM scores at baseline and at 6 weeks are presented in Table 2. As it can be seen, scores of disability and pain intensity have shown reduction in both groups. Whereas, lumbar ROM has shown increase in both groups. In group 1, statistically significant improvements in functional disability, pain intensity and lumbar ROM were observed compared to baseline scores $(P<.05)$. In group 2 , disability, pain intensity, and lumbar ROM parameters were also improved $(P<.05)$.

The analyses of covariance did not reveal significant differences between the 2 groups on reduction of functional disability $\left(\mathrm{F}_{1,67}=6.9, P=.21\right)$ and pain intensity $\left(\mathrm{F}_{1,67}=6.12, P=.41\right)$. Also, there have not been differences in increase of lumbar flexion ROM $\left(\mathrm{F}_{1,67}=3.47, P=.81\right)$ and lumbar extension $\operatorname{ROM}\left(\mathrm{F}_{1,67}=4.2, P=.16\right)$.

\section{Discussion}

The author compared the results obtained before and after 12 treatment sessions using two different modalities in patients with chronic LBP. There were no significant differences between the effects of these modalities in patients with chronic LBP. However, there were statistically significant differences in decreasing functional disability, pain and increasing lumbar ROM in each group. The observed improvements in each group were most likely attributable to the intervention modality.

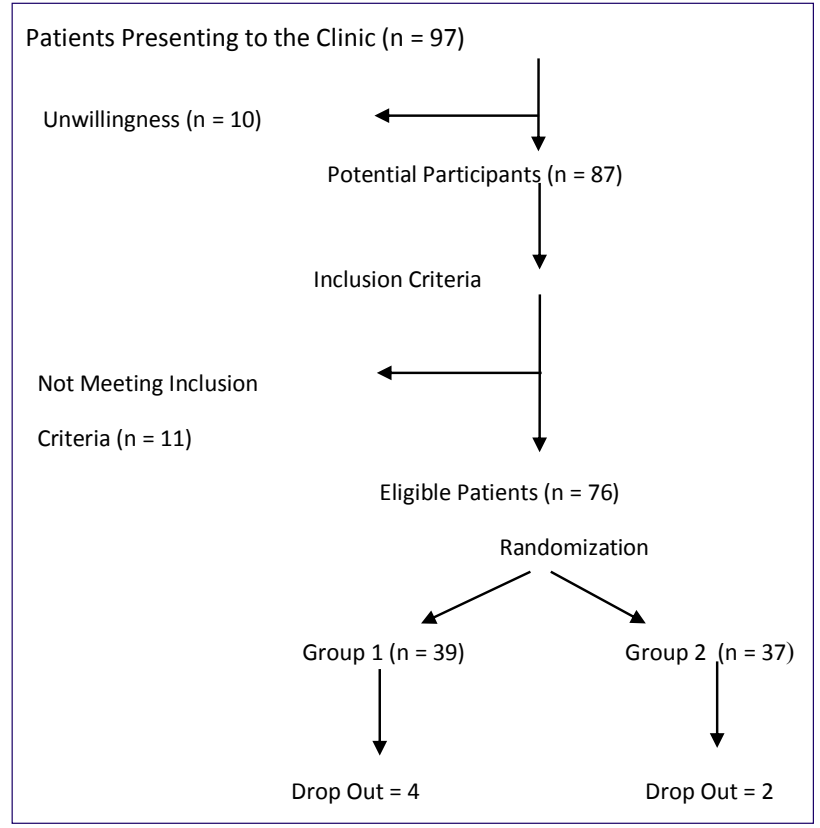

Figure 1. Flow chart detailing the study
The author measured pain perception, physical function and lumbar ROM to get a thorough picture of the outcome of the interventions. These measures are valid and reliable tools to measure progress in patients with chronic LBP. $^{22-24}$

In group 1, participants experienced improvements in functional disability, pain and lumbar ROM in response to MIPE treatment. There has been limited research in using MIPE in patients with musculoskeletal disorders. Hsieh studied the effects of MIPE in patients with knee osteoarthritis. ${ }^{25}$ They conducted a randomized placebo-controlled study. Seventy-three participants received six 40-minute sessions of active or placebo MIPE treatment over the knee joints for 4 weeks. Energy had a wavelength of $890 \mathrm{~nm}$, power of $6.24 \mathrm{~W}$, energy density of 2.08 $\mathrm{J} / \mathrm{cm}^{2} / \mathrm{min}$ and total energy received was $83.2 \mathrm{~J} / \mathrm{cm}^{2}$. They assessed the subjects according to the International Classification of Functioning, Disability, and Health structure. They failed to find any effect of MIPE on pain, daily living activities, function, quality of life, function in sports and recreation.

MIPE may reduce pain by promoting release of nitric oxide in the endothelium as well as heat generation. ${ }^{26} \mathrm{Ex}-$ posure to various wavelengths of energy enhances release of nitric oxide from the hemoglobin and the surrounding tissues. ${ }^{27,28}$ Nitric oxide relaxes smooth muscle cells in the arteries, veins, capillaries and lymph vessels and results in vasodilatation of the blood vessels and thus increases circulation..$^{27,28}$ MIPE treatment increases local blood flow by $400 \%$ after a $20-30$ minute treatment. This was shown by use of a scanning laser Doppler (SLD; Moor Instruments Ltd, UK). ${ }^{10}$ Phototherapy, which includes MIPE, elicits changes in cell membrane permeability, resulting in enhanced synthesis of endorphins, increases nerve cell potential and the resulting pain relief. ${ }^{27,28}$ Thus, release of transiently produced nitric oxide reduces pain and increases function.

The benefits of the MIPE rely on skin contact, pulsation, wavelength, radiant power, and energy density. ${ }^{21}$ The MIPE delivers pulsed adjustable radiant power of up to $10 \mathrm{~mW}$ per diode. It has a power density per diode array of up to $10 \mathrm{~mW}$ per $\mathrm{cm}^{2}$, and an energy density of up to 1.6 joules $/ \mathrm{cm}^{2} / \mathrm{min} .{ }^{21}$ Treatment lasts for 30 minutes delivering up to 48 joules $/ \mathrm{cm}^{2}$ provided that the diodes are in direct contact with the epidermis. ${ }^{21}$

The improvements of the dependent variables in participants of the second group which received LLLT were in agreement with the findings of $\mathrm{Hsieh}$ and $\mathrm{Lee}^{29}$ and Alayat et al. ${ }^{30} \mathrm{Hsieh}$ and Lee ${ }^{29}$ investigated the effects of laser with $890-\mathrm{nm}$ light therapy in 38 patients with chronic LBP. The patients received 40 minutes sessions of hot-pack plus active or placebo $890 \mathrm{~nm}$ light therapy over the lower back 3 times a week for 2 weeks. They reported significant reductions in fear-avoidance beliefs regarding physical activity $(P=.040)$ and work $(P=.007)$ and in the severity of disability $(P=.021)$. Therefore, they concluded that laser may be an effective modality for patients with chronic LBP. Alayat and colleagues ${ }^{30}$ compared the effect of high-inten- 
Table 1. Baseline Characteristics of Patients With Chronic LBP in Both Groups

\begin{tabular}{|c|c|c|c|c|c|}
\hline \multirow[t]{2}{*}{ Parameter } & \multicolumn{2}{|c|}{$\begin{array}{l}\text { MIPE } \\
n=35\end{array}$} & \multicolumn{2}{|c|}{$\begin{array}{c}\text { LLLT } \\
n=35\end{array}$} & \multirow[t]{2}{*}{$P$ Vale $^{a}$} \\
\hline & Mean & SD & Mean & SD & \\
\hline Age $(y)$ & 38.4 & 10.3 & 42.1 & 12.8 & .08 \\
\hline Onset since first episode (y) & 4.6 & 3.9 & 6.1 & 4.2 & .07 \\
\hline BMI & 26.4 & 4.5 & 25.2 & 4.1 & .42 \\
\hline Sex & \multicolumn{2}{|c|}{$60 \%$ female $-40 \%$ male } & \multicolumn{2}{|c|}{$66 \%$ female $-34 \%$ male } & \\
\hline
\end{tabular}

Abbreviations: LBP, low back pain; MIPE, monochromatic infrared photo energy; LLLT, low level laser therapy; BMI, body mass index.

a $P$ values are for baseline differences between the 2 groups. Significance level $\leq .05$.

Table 2. Means and Standard Deviations of Disability, Pain Intensity and Lumbar ROM at Baseline and After 12 Treatment Sessions in Both Groups

\begin{tabular}{|c|c|c|c|c|c|c|}
\hline \multirow{2}{*}{ Parameter } & \multicolumn{3}{|c|}{ Monochromatic* } & \multicolumn{3}{|c|}{ Laser } \\
\hline & Before treatment & After 12 sessions & $P$ Vale & Before treatment & After 12 sessions & $P$ Vale \\
\hline Disability & $41.5 \pm 12.5$ & $20.4 \pm 5.7$ & $.001 *$ & $45.9(14.6)$ & $33.1(11.3)$ & $.001^{*}$ \\
\hline Pain & $44.2 \pm 13.7$ & $28.6 \pm 11.7$ & $.012 *$ & $46.1(14.2)$ & $31.2(12.1)$ & $.021 *$ \\
\hline Flexion ROM & $48.8 \pm 19.4$ & $61.3 \pm 12.6$ & $.02 *$ & $55.3(16.8)$ & $69.5(13.6)$ & $.013^{*}$ \\
\hline Extension ROM & $19.4 \pm 8.2$ & $33.62 \pm 5.6$ & $.01^{*}$ & $22.6(9.6)$ & $32.1(9.3)$ & $.03 *$ \\
\hline
\end{tabular}

Abbreviations: ROM, range of motion.

sity laser therapy (HILT), alone or combined with physical exercise, in patients with chronic LBP. They randomly assigned 72 patients into 3 groups treated with HILT and exercise, placebo laser and exercise, and HILT alone in groups 1,2 , and 3 , respectively. Outcomes measures included lumbar ROM, VAS, the Roland disability questionnaire and the modified Oswestry disability questionnaire. They reported that HILT and exercise are more effective in patients with chronic LBP than either HLLT alone or placebo laser with exercise.

On the other hand, the improvements of the dependent variables in participants of the second group who received LLLT did not agree with the findings of Glazov et al. ${ }^{31}$ Glazov et al reported that sham and the laser groups had no significant difference in pain or functional disability at 6 weeks in patients with chronic LBP.

van Middelkoop et al ${ }^{32}$ studied all interventions available for the chronic LBP. They have shown that multiple interventions have better results compared to single-agent interventions. This study shows that combining MIPE or LLLT with physical exercise may be the most effective way to reduce pain and improve function and Lumbar ROM in patients with chronic LBP.

There are a number of potential biases that could threaten the validity or the conclusions and for these reasons future investigation remains necessary in order to better understand the clinical value of the MIPE in the management of chronic LBP. Perhaps the biggest limitation of this study relates to the lack of control group. Other limitations of the study include its small sample size and limited duration of follow up. Patients were advised not to receive other forms of physical therapy and pain medications during the trial. However, their adherence to these instructions could not be controlled. Therefore, there is a small possibility that both groups may have improved due to time alone and avoidance of vigorous activity throughout the treatment time. This study had multiple exclusion criteria that may have reduced the external validity of this trial.
Further studies on the effects of MIPE and LLLT using different settings for power, wavelength, energy density, and treatment duration may be needed. Still further, additional research is needed to more precisely identify the role that nitric oxide plays in the outcomes and whether or not the improvement in the dependent variables that was observed in this study is sustained in the long-term. Based on the results of this randomized clinical trial, the MIPE and LLLT may be equally effective in decreasing pain and increasing physical function in patients with chronic LBP.

\section{Conflict of Interest}

The author has no conflict of interest to declare.

\section{References}

1. Cho KH, Beom JW, Lee TS, Lim JH, Lee TH, Yuk JH. Trunk muscles strength as a risk factor for nonspecific low back pain: a pilot study. Ann Rehabil Med. 2014;38(2):234-240. doi:10.5535/arm.2014.38.2.234

2. Pivec R, Stokes M, Chitnis AS, Paulino CB, Harwin SF, Mont MA. Clinical and economic impact of TENS in patients with chronic low back pain: analysis of a nationwide database. Orthopedics. 2013;36(12):922928. doi:10.3928/01477447-20131120-04

3. Atalay NS, Sahin F, Atalay A, Akkaya N. Comparison of efficacy of neural therapy and physical therapy in chronic low back pain. Afr J Tradit Complement Altern Med. 2013;10(3):431-435. doi:10.4314/ajtcam. v10i3.8

4. Murtezani A, Hundozi H, Orovcanec N, Sllamniku S, Osmani T. A comparison of high intensity aerobic exercise and passive modalities for the treatment of workers with chronic low back pain: a randomized, controlled trial. Eur J Phys Rehabil Med. 2011; 47(3):359-366.

5. Thiese MS, Hughes M, Biggs J. Electrical stimulation for chronic non-specific low back pain in a working-age population: a 12-week double blinded 
randomized controlled trial. BMC Musculoskelet Disord. 2013;14:117. doi:10.1186/1471-2474-14-117

6. Hill C. Is yoga an effective treatment in the management of patients with chronic low back pain compared with other care modalities - a systematic review? J Complement Integr Med. 2013;10(1):211219. doi:10.1515/jcim-2012-0007

7. Barbosa AC, Martins FL, Barbosa MC, Dos Santos RT. Manipulation and selective exercises decrease pelvic anteversion and low-back pain: a pilot study. $J$ Back Musculoskelet Rehabil. 2013;26(1):33-36.

8. Ansari NN, Naghdi S, Naseri N, et al. Effect of therapeutic infra-red in patients with non-specific low back pain: a pilot study. J Bodyw Mov Ther. 2014;18(1):75-81. doi:10.1016/j.jbmt.2013.05.014

9. Bahrami-Taghanaki $\mathrm{H}$, Liu $\mathrm{Y}$, Azizi $\mathrm{H}$, et al. A randomized controlled trial of acupuncture for chronic low back pain. J Altern Complement Med. 2014; 20(5):A40. doi:10.1089/acm.2014.5101.abstract

10. Burke T. Questions and answers about the MIRE treatment. Adv Skin Wound Care. 2003;12:369-371. doi:10.1097/00129334-200312000-00016

11. Ammar TA. Monochromatic Infrared Photo Energy in Diabetic Peripheral Neuropathy. ISRN Rehabilitation. 2012;2012: 484307. doi:10.5402/2012/484307

12. Ammar TA. Monochromatic Infrared Photo Energy versus low level laser therapy in patients with knee osteoarthritis. J lasers Med Sci. 2014;5(4):176-182.

13. Mitchell U, Myrer JW, Johnson AW, Hilton SC. Restless leg syndrome and near-infrared light: An alternative treatment option. Physiother Theory Pract. 2010;15(3):1-7. doi:10.3109/09593985.2010.511440

14. Dogan SK, Ay S, Evcik D. The effectiveness of low laser therapy in subacromial impingement syndrome: a randomized placebo controlled double-blind prospective study. Clinics (Sao Paulo). 2010;65(10):1019-1022.

15. Jang H, Lee H. Meta-analysis of pain relief effects by laser irradiation on joint areas. Photomed Laser Surg. 2012;30(8):405-417. doi:10.1089/pho.2012.3240

16. Salmos-Brito JA, de Menezes RF, Teixeira CE, et al. Evaluation of low-level laser therapy in patients with acute and chronic temporomandibular disorders. Lasers Med Sci. 2013;28(1):57-64. doi:10.1007/ s10103-012-1065-8

17. Rayegani S, Bahrami M, Samadi B, Sedighipour L, Mokhtarirad M, Eliaspoor D. Comparison of the effects of low energy laser and ultrasound in treatment of shoulder myofascial pain syndrome: a randomized single-blinded clinical trial. Eur J Phys Rehabil Med. 2011;47(3); 381-389.

18. Alghadir A, Omar MT, Al-Askar AB, Al-Muteri NK. Effect of low-level laser therapy in patients with chronic knee osteoarthritis: a single-blinded randomized clinical study. Lasers Med Sci. 2014;29(2): 749-755. doi:10.1007/s10103-013-1393-3

19. Ay S, Doğan SK, Evcik D. Is low-level laser therapy effective in acute or chronic low back pain? Clin
Rheumatol. 2010;29(8):905-910.

20. Basford JR, Sheffield CG, Harmsen WS. Laser therapy: a randomized, controlled trial of the effects of lowintensity Nd:YAG laser irradiation on musculoskeletal back pain. Arch Phys Med Rehabil. 1999; 80(6):647652. doi:10.1016/s0003-9993(99)90167-3

21. Belanger AY. Evidence-Based Guide to Therapeutic Physical Agents. Philadelphia, PA: Lippincott Williams \& Wilkins; 2002:191-221.

22. Ceran F, Ozcan A. The relationship of the Functional Rating Index with disability, pain, and quality of life in patients with low back pain. Med Sci Monit. 2006;12(10):CR435-CR439.

23. Zampelis V, Ornstein E, Franzén H, Atroshi I. A simple visual analog scale for pain is as responsive as the WOMAC, the SF-36, and the EQ-5D in measuring outcomes of revision hip arthroplasty. Acta Orthop. 2014;85(2):128-132. doi:10.3109/17453674.2014.887 951

24. Williams R, Binkley J, Bloch R, Goldsmith CH, Minuk T. Reliability of the modified-modified Schober and double inclinometer methods for measuring lumbar flexion and extension. Phys Ther. 1993;73(1):33-44.

25. Hsieh R, Lo MT, Lee W, Liao W. Therapeutic effects of short-term monochromatic infrared energy therapy on patients with knee osteoarthritis: a double-blind, randomized, placebo-controlled study. J Orthop Sports Phys Ther. 2012;42(11):947-956.

26. Matsunaga, K, Furchgott, RF. Interactions of light and sodium nitrite in producing relaxation of rabbit aorta. J Pharmacol Exp Ther. 1989;248(2):687-695.

27. Abramson SB. Osteoarthritis and nitric oxide. Osteoarthr Cartil. 2008;16(suppl 2):S15-S20.

28. Hancock CM, Riegger-Krugh C. Modulation of pain in osteoarthritis: the role of nitric oxide. Clin J Pain. 2008;24(4):353-365. doi:10.3109/17453674.201 4.887951

29. Hsieh R, Lee WC. Short-term therapeutic effects of 890-nanometer light therapy for chronic low back pain: a double-blind randomized placebocontrolled study. Lasers Med Sci. 2014;29(2):671-679. doi:10.1007/s10103-013-1378-2

30. Alayat MS, Atya AM, Ali MM, Shosha TM. Longterm effect of high-intensity laser therapy in the treatment of patients with chronic low back pain: a randomized blinded placebo-controlled trial. Lasers Med Sci. 2014;29(3):1065-1073. doi:10.1007/s10103013-1472-5

31. Glazov G, Yelland, M, Emery J. Low-dose laser acupuncture for non-specific chronic low back pain: a double-blind randomized controlled trial. Acupunct Med. 2014;32(2):116-123. doi:10.1136/ acupmed-2013-010456

32. van Middelkoop M, Rubinstein SM, Kuijpers T, et al. A systematic review on the effectiveness of physical and rehabilitation interventions for chronic nonspecific low back pain. Eur Spine J. 2011;20(1):19-39. doi:10.1007/s00586-010-1518-3 